giv

$$
\left(1-e^{i \theta}\right) \mathbf{r}=\mathbf{s}_{n}, \quad \text { or } \quad \mathbf{r}=\frac{1}{2 \sin \frac{1}{2} \theta} e^{i(\pi-\theta) / 2} \mathbf{s}_{n}
$$

This means that the point $O$ is the vertex of the isosceles triangle whose base is $O^{\prime} O_{n}$ and whose angle at $O$ is $\theta$ if $\theta<\pi$ and is $2 \pi-\theta$ if $\theta>\pi$. In the former case the sense $O^{\prime} O_{n} O$ is positive, in the latter it is negative. If $\theta=\pi$, the resultant displacement is a reversal (Umwendung) about the midpoint of $O^{\prime} O_{n}$. If $\theta \equiv 0(\bmod 2 \pi)$ the resultant displacement reduces to the translation $\mathrm{s}_{n}$.

If, in particular, the angles of rotation $\theta^{\prime \prime}, \theta^{\prime \prime \prime}, \cdots, \theta^{(n)}$ are equal, respectively, to the exterior angles of the polygon of centers at $O^{\prime \prime}, O^{\prime \prime \prime}, \cdots, O^{(n)}$, the vector $\mathrm{s}_{n}$ has, as appears at once from its construction (Fig. 3), the direction and sense of $\mathbf{c}_{n}$ and a length equal to the perimeter of the polygon $O^{\prime} O^{\prime \prime} \ldots O^{(n)}$. If, in addition, $\theta^{\prime}$ is equal to the exterior angle at $O^{\prime}$ and the polygon is convex, the resultant rotation is zero, and the resultant displacement reduces to the translation $\mathbf{s}_{n}$.

ANN ARBor, December, 1907.

\title{
ON AN INTEGRAL APPEARING IN PHOTOMETRY.
}

BY PROFESSOR A. S. CHESSIN.

(Read before the Southwestern Section of the American Mathematical Society, November 30, 1907.)

In the course of research on the variation of the intensity of illumination, Mr. E. P. Hyde found it necessary to evaluate the integral

$$
\int \frac{i \cos \phi \cos \theta}{r^{2}} d S
$$

taken over a part of the surface of a cylinder. $\dagger$ Having encountered some difficulties on the mathematical side of the problem, Mr. Hyde requested the author to compute the value of this integral. As this computation contains some features of interest to mathematicians the solution is here presented

† "Talbot's law as applied to the rotating sectored disc," by E. P. Hyde, Bulletin of the U. S. Bureau of Standards, vol. 2 , No. 1. 
without, however, referring to the physical aspects of the problem. The purely mathematical problem, then, may be stated as follows :

Evaluate the integral

$$
J=i a \int_{-h}^{h} d y \int_{-\cos ^{-1} a / l}^{\cos ^{-1} a / l} \frac{(\alpha-l \cos \alpha)(l-a \cos \alpha) d \alpha}{\left(a^{2}+l^{2}-2 a l \cos \alpha+y^{2}\right)^{2}} .
$$

By means of the substitutions

$$
\begin{array}{ll}
\tan \frac{1}{2} \alpha=x, & \frac{(l+a)^{2}+y^{2}}{(l-a)^{2}+y^{2}}=z, \\
\frac{l+a}{l-\alpha}=p, & \frac{(l+a)^{2}+h^{2}}{(l-a)^{2}+h^{2}}=q,
\end{array}
$$

the given integral is transformed into

$$
i\left(1-\frac{a}{l}\right) \int_{p^{2}}^{q} \sqrt{\frac{z-1}{p^{2}-z}} d z \int_{0}^{1 / \sqrt{p}} \frac{\left(p^{2} x^{4}-1\right) d x}{\left(x^{2}+1\right)\left(z x^{2}+1\right)^{2}}
$$

and, after effecting the integration with regard to $x$, becomes

where

$$
i(1-a / l)\left(J_{1}+J_{2}+J_{3}\right)
$$

$$
\begin{aligned}
& J_{1}=\left(p^{2}-1\right) \tan ^{-1} \frac{1}{\sqrt{p}} \int_{p^{2}}^{q} \frac{d z}{(z-1) \sqrt{(z-1)\left(p^{2}-z\right)}}, \\
& J_{2}=\frac{1}{2} \sqrt{p} \int_{p^{2}}^{q} \frac{(p-z) d z}{z \sqrt{(z-1)\left(p^{2}-z\right)}}, \\
& J_{3}=\int_{p^{2}}^{q}\left[\frac{z-p^{2}}{z-1}-\frac{p^{2}+z^{2}}{2 z}\right] \frac{\tan ^{-1} \sqrt{z / p} d z}{\sqrt{z(z-1)\left(p^{2}-z\right)}} .
\end{aligned}
$$

The integrals $J_{1}$ and $J_{2}$ are readily evaluated by the ordinary rules. The third integral $J_{3}$ apparently offers some difficulties. The integration, however, is very simple and does not even require the introduction of elliptic functions.

In fact, consider the integral

$$
U=\int\left[\frac{z-p^{2}}{z-1}-\frac{p^{2}+z^{2}}{2 z}\right] \frac{d z}{\sqrt{z(z-1)\left(p^{2}-z\right)}} .
$$

It belongs to a class of pseudo-elliptic integrals discussed by 
Euler,* Raffy, $\dagger$ and Goursat. $\$$ Adopting Goursat's method, we have in this case three relations of involution by which the roots of the equation $z(z-1)\left(p^{2}-z\right)=0$ are permuted in pairs. Two of these relations are

$$
z^{\prime} z^{\prime \prime}=p^{2}, \quad z^{\prime} z^{\prime \prime}-\left(z^{\prime}+z^{\prime \prime}\right)+p^{2}=0 .
$$

On the other hand, if we put

where

$$
\frac{z-p^{2}}{z-1}-\frac{p^{2}+z^{2}}{2 z}=f_{1}(z)+f_{2}(z)
$$

$$
f_{1}(z)=\frac{z-p^{2}}{z-1}-z, \quad f_{2}(z)=\frac{1}{2}\left(z-\frac{p^{2}}{z}\right)
$$

we note that each one of the functions $f_{1}$ and $f_{2}$ satisfies a relation, corresponding to one or the other of the two relations of involution given above, viz.,

$$
f_{1}(z)+f_{1}\left(\frac{p^{2}-z}{1-z}\right) \equiv 0, \quad f_{2}(z)+f_{2}\left(\frac{p^{2}}{z}\right) \equiv 0 .
$$

Hence, $\S$ the integral $U$ is pseudo-elliptic.

At the same time, if we apply the author's reduction formulas given in the pages of this Bulletin, || viz., formulas (5) and (6), where we should put $m=r=3, \Sigma n_{k}=s=\rho=2$, and, consequently, $q=1$, we find that

$$
\begin{aligned}
U=\frac{(A z+B) \sqrt{z(z-1)\left(p^{2}-z\right)}}{z(z-1)} \\
+\lambda_{0} \int \frac{d z}{\sqrt{z(z-1)\left(p^{2}-z\right)}}+\lambda_{1} \int \frac{z d z}{\sqrt{z(z-1)\left(p^{2}-z\right)}} \cdot
\end{aligned}
$$

But the two integrals on the right-hand side are obviously elliptic. Hence, since it has been shown that $U$ is pseudo-

* "Sur une formule d'Euler," par M. Hermite, Journal de Liouville, 1880. † "Sur les transformations invariantes des différentielles elliptiques," par L. Raffy, Bulletin de la Soc. Math. de France, 1884.

† "Sur les integrales pseudo-elliptiques," par E. Goursat, Bulletin de la Soc. Math. de France, 1885. See also Goursat, Cours d'analyse, vol. 1, pp. 264-266.

\& L. c.

I| "Note on hyperelliptic integrals," Bulletis, vol. 4, pp. 93-96. 
elliptic, we must have $\lambda_{0}=\lambda_{1}=0$, so that, barring a constant of integration,

$$
U=(A z+B) \sqrt{\frac{p^{2}-z}{z(z-1)}} .
$$

It now only remains to determine the coefficients $A$ and $B$. This is readily done by comparing both sides of the identity

$$
\frac{z-p^{2}}{z-1}-\frac{p^{2}+z^{2}}{2 z}=\sqrt{z(z-1)\left(p^{2}-z\right)} \frac{d}{d z}\left[(A z+B) \sqrt{\frac{p^{2}-z}{z(z-1)}}\right]
$$

which gives $A=B=1$ and, therefore, barring the constant of integration,

$$
U=(z+1) \sqrt{\frac{p^{2}-z}{z(z-1)}} .
$$

Returning now to the integration of $J_{3}$ we observe that

$$
\begin{aligned}
J_{3} & =\int_{p^{2}}^{q} \tan ^{-1} \sqrt{\frac{z}{p}} d U=\left[U \tan ^{-1} \sqrt{\frac{z}{p}}\right]_{p^{2}}^{q}-\frac{1}{2} \sqrt{p} \int_{p^{2}}^{q} \frac{U d z}{(z+p) \sqrt{z}} \\
& =(q+1) \sqrt{\frac{p^{2}-q}{q(q-1)}} \tan ^{-1} \sqrt{\frac{q}{p}}+\frac{1}{2} \sqrt{p} \int_{p^{2}}^{q} \frac{(z+1)\left(z-p^{2}\right) d z}{z(z+p) \sqrt{(z-1)\left(p^{2}-z\right)}}
\end{aligned}
$$

or, adding the integral $J_{2}$,

$$
\begin{aligned}
J_{2}+J_{3}=(q+1) & \sqrt{\frac{p^{2}-q}{q(q-1)}} \tan ^{-1} \sqrt{\frac{q}{p}} \\
& -\frac{1}{2}\left(p^{2}-1\right) \sqrt{p} \int_{p^{2}}^{q} \frac{d z}{(z+p) \sqrt{(z-1)\left(p^{2}-z\right)}}
\end{aligned}
$$

It is a simple matter now to complete the integration. The result is *

$$
\begin{aligned}
J=\frac{i}{l}\left\{2 a \tan ^{-1} \frac{h}{\sqrt{l^{2}-a^{2}}}+h\left(\sqrt{q}+\frac{1}{\sqrt{q}}\right) \tan ^{-1} \sqrt{\frac{q}{p}}\right. \\
\left.-2 h \cot ^{-1} \sqrt{p}\right\} .
\end{aligned}
$$

\section{SaINT Louis,} November 30, 1907.

\footnotetext{
* It is given in Mr. Hyde's paper in a slightly different but equivalent form. L. c., p. 8 .
} 